\title{
Evaluación de la calidad en la atención al usuario del servicio de urgencias del hospital público de Yopal en Casanare, Colombia
}

\author{
Assessment of the user service quality of the emergency department in the \\ public hospital of Yopal, Casanare, Colombia
}

Título abreviado: Evaluación de la calidad en la atención al usuario

Aradeisy Ibarra Picón ${ }^{1}$ Edwin Rua Ramirez ${ }^{2}$

\section{Resumen}

Objetivo. En esta investigación se evalúa la calidad en el proceso de atención al usuario del servicio de urgencias del hospital público de Yopal en Casanare, Colombia. Metodología. Esta investigación es de tipo descriptivo, exploratorio, apoyada en varias técnicas de investigación, iniciando con un cuestionario-encuesta, donde se realiza un análisis cuantitativo y cualitativo de la calidad de atención al usuario, la capacidad de la planta física, la comodidad, seguridad y confiabilidad del servicio; además se aplica la técnica de observación directa, análisis e interpretación de fenómenos, acontecimientos y hechos que prevalecen en el área. Resultados. Con este proyecto se pudo determinar la satisfacción o conformidad de los usuarios del servicio de urgencias y la aplicación de los protocolos de atención bajo estándares de calidad, cuál es el trato dado por los trabajadores y profesionales de la salud hacia los usuarios, las necesidades de capacitación al personal de atención, y posibles mejoras o modificaciones a los protocolos, cambios o recomendaciones para mejorar la calidad de atención. Conclusiones. El Hospital de Yopal no tiene capacidad suficiente en el área de urgencias para atender la alta demanda proveniente de los habitantes de Yopal y los municipios aledańos, la constante queja de los usuarios que dicen ser mal atendidos, el supuesto incumplimiento en su labor hacia los enfermos y familiares en la mayoría de las áreas del hospital, falta de personal profesional para la atención en el servicio de urgencias, largas jornadas de espera de los usuarios enfermos.

Palabras claves: atención al usuario, calidad, protocolos, servicio de urgencias, hospital público.

\section{Abstract}

Objective. In this research is evaluated the quality in the customer service of the emergency department of the public Hospital of Yopal, Casanare, Colombia. Method. This investigation is of descriptive, exploratory type, supported on several investigation techniques, starting with a questionnaire - survey where there is carried out a quantitative and qualitative analysis of the quality of attention to the user, the capacity of the physical plant, comfort, safety and reliability of the service; besides, It is applied the technique of direct observation, analysis and interpretation of phenomena, events and facts that prevail in the area. Results. With this project it was possible to determine the satisfaction or conformity of the users of the first aid service and the application of the protocols of attention under quality standards, which is the treatment given by workers and 
health professionals toward users, if it is necessary to qualify the attention staff, if it is necessary to improve or modify the protocols, if changes or recommendations should be made to improve the quality of the attention. Conclusions. Yopal Hospital does not have enough capacity in the Emergency Department to meet the high demand coming from Yopal inhabitants and the surrounding municipalities, the incessant complaint of users claiming to be supposedly poorly served, the supposed nonperformance in its work towards the patients and relatives in most of the areas of the Hospital, lack of professional staff for the attention in the emergency department, long days of waiting for sick users.

Keywords: User service, quality, protocols, emergency department, public hospital.

\section{Introducción}

Los servicios de urgencias del hospital público de Yopal, Casanare, están viviendo una problemática compleja. Aunque es una situación extensa en todo el país, preocupa excesivamente que Casanare no cuente con una red alterna de apoyo ante las crisis de emergencias, dejando un aire de intranquilidad ya que se presentan falencias en aspectos importantes $(1-6)$.

El objetivo de este trabajo es determinar el grado de conformidad e inconformidad de los usuarios en relación a la atención prestada en el servicio de urgencias del hospital de Yopal. Conjuntamente, aplicar las nuevas estrategias gerenciales, apoyados en la bibliografía con el fin de mejorar la atención en el servicio de urgencias del hospital de Yopal.

Los resultados obtenidos fueron presentados ante los directivos y administrativos del hospital y ante la comunidad científica. Como resultado práctico se busca mejorar la atención al usuario en el hospital apoyado en un modelo de gestión con estándares de calidad y protocolos y así reducir las quejas de los usuarios o enfermos que llegan a diario al hospital público de Yopal.

\section{Materiales y métodos}

Esta investigación es de tipo descriptivo y permite identificar la calidad en la prestación del servicio de urgencias del hospital de Yopal. El enfoque o tipo de investigación es cualitativa y cuantitativa (.

Es cualitativa a través de entrevistas con los expertos y la observación que permitirá analizar y explorar los fenómenos en profundidad. El análisis cualitativo utiliza la recolección de datos sin medición numérica para descubrir o afinar preguntas de investigación en el proceso de interpretación.

Con las encuestas aplicadas a los usuarios se puede identificar la calidad en la prestación del servicio de urgencias del hospital de Yopal de manera cuantitativa (6-11). La interpretación constituye una explicación de cómo los resultados encajan en el conocimiento existente (12). En una investigación cuantitativa se pretende generalizar los resultados encontrados en un grupo o segmento (muestra) a una colectividad mayor (universo o población) (1). También se busca que los estudios efectuados puedan replicarse.

\section{Variables:}

- Calidad del servicio: estándares superiores que le permitan al usuario y su familia estar satisfechos con la atención dispensada.

- Planta física: consta del espacio exterior y el interior.

- Personal de atención: personas que ofrece el hospital para prestar un servicio amable al cliente.

- Comodidad del servicio: facilita la estancia en el lugar o la realización de ciertas actividades. 
- Seguridad en el servicio: ausencia de riesgo y confianza en la atención prestada.

- Confiabilidad del servicio: capacidad del hospital y el personal de atención, para desempeñar las funciones requeridas, en las condiciones establecidas.

- Inconformidad: no conforme. Añoranza por algo mejor que la situación actual

\section{Muestra}

Para la determinación del tamaño de la muestra se emplearon características del muestreo aleatorio simple para poblaciones infinitas (13-16), dado que este tipo de muestreo permite determinar el número de encuestas teniendo en cuenta los siguientes aspectos:

Caso: población infinita cualitativa. Distribución proporcional, de acuerdo a los predios objeto de estudio, con el objeto de tener una muestra con características lo suficientemente representativas para el estudio (17-18).

Cálculo del tamaño de muestra empleando un 92 $\%$ del nivel confianza y un margen de error del 8 $\%$.

$$
n=\frac{Z^{2} P(1-P)}{e^{2}}
$$

Donde

Z: Margen de confiabilidad (expresado en desviaciones estándar).

P: Probabilidad de que el evento ocurra (expresado en unidad).

(1-P): Probabilidad de que el evento no ocurra.

e: Error de estimación (máximo error permisible por unidad) Con base en lo anterior se definieron muestrear 120 personas atendidas por el servicio de urgencias del hospital de Yopal.

\section{Instrumentos de medición y técnicas}

Para esta investigación se aplicó un instrumento (cuestionario-encuesta) tipo escalamiento Likert. Se realizaron entrevista a expertos y a través de la observación se pudo analizar y explorar los fenómenos en profundidad (19-22).

\section{Validación del cuestionario}

La validación del cuestionario se hizo a través de prueba piloto y validación por expertos.

Figura 1. Cuestionario-encuesta diseñado para la investigación.

\section{ENCUESTA DE ATENCIÓN AL USUARIO EN EL SERVICIO DE URGENCIAS DEL HOSPITAL PÚBLICO DE YOPAL, CASANARE.}

PROYECTO: Evaluación de la calidad en la atención al usuario del servicio de urgencias del hospital público de Yopal, Casanare-Colombia.

Por favor lea cuidadosamente las siguientes preguntas y responda marcando con una X la respuesta que considere conveniente, agradecemos su colaboración dando respuestas sinceras a las interrogantes. 


\begin{tabular}{|c|c|c|c|c|c|c|}
\hline & & 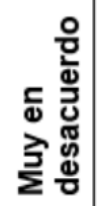 &  & 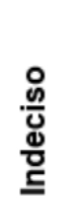 & 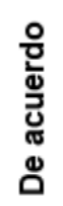 & 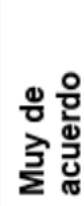 \\
\hline \multicolumn{7}{|c|}{ Planta fisica } \\
\hline 1 & $\begin{array}{l}\text { El servicio de Urgencias tiene una planta física } \\
\text { adecuada para brindar el servicio. }\end{array}$ & & & & & \\
\hline 2 & $\begin{array}{l}\text { El servicio de Urgencias cuenta con tecnología y } \\
\text { equipos apropiados para la atención médica. }\end{array}$ & & & & & \\
\hline 3 & $\begin{array}{l}\text { El servicio de urgencias tiene capacidad física } \\
\text { suficiente para la atención de la población de } \\
\text { Yopal. }\end{array}$ & & & & & \\
\hline \multicolumn{7}{|c|}{ Personal de atención } \\
\hline 4 & $\begin{array}{l}\text { La cantidad de personal en atención de } \\
\text { urgencias es el apropiado }\end{array}$ & & & & & \\
\hline 5 & $\begin{array}{l}\text { El personal de atención tiene preferencias sobre } \\
\text { algunos pacientes. }\end{array}$ & & & & & \\
\hline 6 & $\begin{array}{l}\text { La atención en la recepción de urgencias es } \\
\text { excelente. }\end{array}$ & & & & & \\
\hline 7 & $\begin{array}{l}\text { La atención brindada por el personal profesional } \\
\text { es muy efectiva. }\end{array}$ & & & & & \\
\hline \multicolumn{7}{|c|}{ Comodidad del servicio } \\
\hline 8 & $\begin{array}{l}\text { El tiempo de atención en urgencias es suficiente } \\
\text { y apropiado. }\end{array}$ & & & & & \\
\hline 9 & $\begin{array}{l}\text { La sala de espera del servicio de urgencias es } \\
\text { cómoda. }\end{array}$ & & & & & \\
\hline 10 & $\begin{array}{l}\text { La climatización del servicio de urgencias es } \\
\text { confortable. }\end{array}$ & & & & & \\
\hline 11 & $\begin{array}{l}\text { La iluminación en el sector de urgencias es muy } \\
\text { buena. }\end{array}$ & & & & & \\
\hline 12 & $\begin{array}{l}\text { La Higiene en el servicio de urgencias es } \\
\text { agradable. }\end{array}$ & & & & & \\
\hline \multicolumn{7}{|c|}{ Seguridad en el servicio } \\
\hline 13 & $\begin{array}{l}\text { El servicio de urgencias cuenta con personal de } \\
\text { seguridad eficaz. }\end{array}$ & & & & & \\
\hline \multicolumn{7}{|c|}{ Confiabilidad del servicio } \\
\hline 14 & $\begin{array}{l}\text { El personal de recepción atiende oportunamente } \\
\text { y con educación a los usuarios. }\end{array}$ & & & & & \\
\hline 15 & 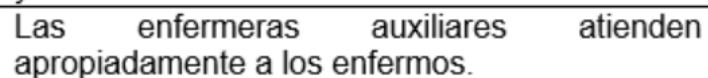 & & & & & \\
\hline 16 & $\begin{array}{l}\text { Las enfermeras Jefes coordinan } \\
\text { adecuadamente la atención de los usuarios. }\end{array}$ & & & & & \\
\hline 17 & $\begin{array}{l}\text { Los médicos están capacitados para atender } \\
\text { enfermedades urgentes. }\end{array}$ & & & & & \\
\hline 18 & $\begin{array}{l}\text { El médico lo escucha con atención antes de } \\
\text { hacer el diagnostico. }\end{array}$ & & & & & \\
\hline
\end{tabular}




\begin{tabular}{|c|l|l|l|l|l|l|}
\hline 19 & $\begin{array}{l}\text { El médico lo examina detalladamente antes de } \\
\text { hacer el diagnostico. }\end{array}$ & & & & \\
\hline 20 & $\begin{array}{l}\text { El médico le solicita exámenes médicos para un } \\
\text { buen diagnóstico en caso de ser necesario. }\end{array}$ & & & & & \\
\hline
\end{tabular}

Fecha de elaboración:

Fuente. Elaboración propia.

\section{Procedimientos}

Para el cálculo de la muestra se utilizó la fórmula de población infinita cualitativa, ya que la población de atención de urgencias varía constantemente. Se aplicaron las encuestas en el servicio de urgencias en diferentes fechas aleatoriamente. El análisis de las encuestas se hizo en Excel y se analizaron los resultados estadísticamente en valor absoluto y porcentaje (23-25).

\section{Validez de apariencia (valoración por ex- pertos)}

Para la validación por expertos se hizo la revisión y aprobación por parte del Doctor Carlos Marcuello, Director de la tesis (Funiber), y algunos profesionales (médicos y enfermeras jefe) del hospital de Yopal.

Figura 2. Fachada del hospital público de Yopal.

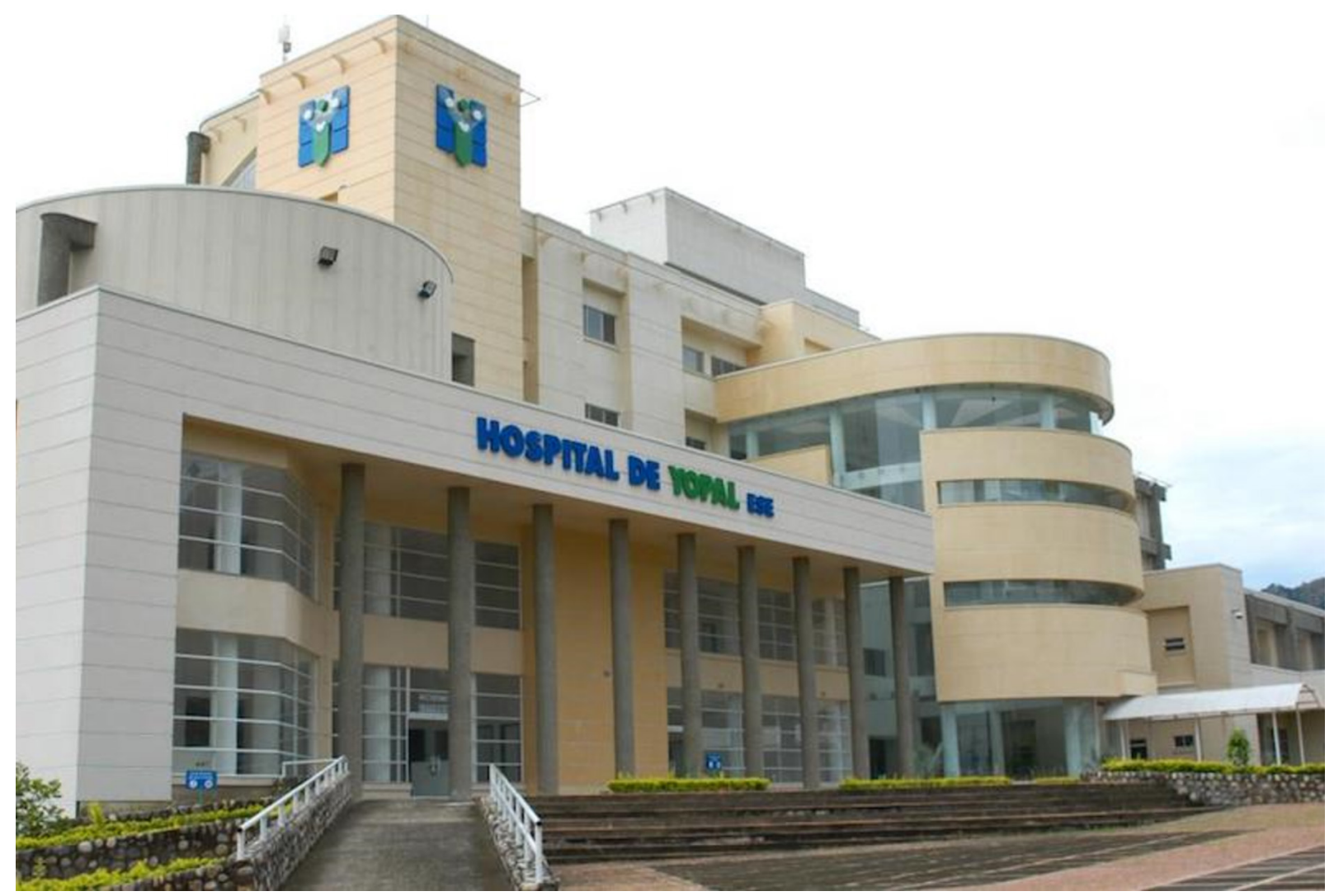

Fuente. Tomada de http://www.violetastereo.com 


\section{Resultados}

Se encuestaron 120 usuarios del servicio de urgencias del hospital público de Yopal, según lo calculado en la muestra. De estas se anularon seis encuestas por tener varias respuestas en una sola pregunta, lo cual no permitía un nivel de confiabilidad. En total se analizaron 114 encuestas, en una gran parte fueron usuarios de Yopal, pero también en algunos casos personas de municipios vecinos.
En la siguiente tabla se muestra el análisis de las encuestas en porcentaje de acuerdo a las respuestas dadas por los usuarios encuestados.

Tabla 3. Caracterización del cuidador familiar.

\begin{tabular}{|c|c|c|c|c|c|}
\hline Aspectos & $\begin{array}{l}\text { Muy en } \\
\text { desacuerdo }\end{array}$ & $\begin{array}{c}\text { En } \\
\text { desacuerdo }\end{array}$ & Indeciso & De acuerdo & $\begin{array}{l}\text { Muy de } \\
\text { acuerdo }\end{array}$ \\
\hline $\begin{array}{l}\text { 1. El servicio de urgencias tiene una planta } \\
\text { física adecuada para brindar el servicio }\end{array}$ & $10 \%$ & $18 \%$ & $15 \%$ & $43 \%$ & $15 \%$ \\
\hline $\begin{array}{l}\text { 2. El servicio de urgencias cuenta con } \\
\text { tecnología y equipos apropiados para la } \\
\text { atención médica }\end{array}$ & $5 \%$ & $14 \%$ & $25 \%$ & $39 \%$ & $17 \%$ \\
\hline $\begin{array}{l}\text { 3. El servicio de urgencias tiene capacidad } \\
\text { física suficiente para la atención de la } \\
\text { población de Yopal. }\end{array}$ & $28 \%$ & $28 \%$ & $24 \%$ & $12 \%$ & $8 \%$ \\
\hline $\begin{array}{l}\text { 4. La cantidad de personal en atención de } \\
\text { urgencias es el apropiado }\end{array}$ & $17 \%$ & $32 \%$ & $25 \%$ & $22 \%$ & $5 \%$ \\
\hline $\begin{array}{l}\text { 5. El personal de atención tiene preferencias } \\
\text { sobre algunos pacientes. }\end{array}$ & $12 \%$ & $34 \%$ & $22 \%$ & $32 \%$ & $11 \%$ \\
\hline $\begin{array}{l}\text { 6. La atención en la recepción de urgencias es } \\
\text { excelente }\end{array}$ & $5 \%$ & $22 \%$ & $24 \%$ & $32 \%$ & $17 \%$ \\
\hline $\begin{array}{l}\text { 7. La atención brindada por el personal } \\
\text { profesional es muy efectiva. }\end{array}$ & $6 \%$ & $17 \%$ & $26 \%$ & $35 \%$ & $16 \%$ \\
\hline $\begin{array}{l}\text { 8. El tiempo de atención en urgencias es } \\
\text { suficiente y apropiado }\end{array}$ & $33 \%$ & $26 \%$ & $15 \%$ & $19 \%$ & $6 \%$ \\
\hline $\begin{array}{l}\text { 9. La sala de espera del servicio de urgencias } \\
\text { es cómoda }\end{array}$ & $21 \%$ & $25 \%$ & $24 \%$ & $18 \%$ & $11 \%$ \\
\hline $\begin{array}{l}\text { 10. La climatización del servicio de urgencias } \\
\text { es confortable }\end{array}$ & $5 \%$ & $17 \%$ & $16 \%$ & $46 \%$ & $16 \%$ \\
\hline $\begin{array}{l}\text { 11. La iluminación en el sector de urgencias } \\
\text { es muy buena }\end{array}$ & $4 \%$ & $4 \%$ & $15 \%$ & $58 \%$ & $20 \%$ \\
\hline $\begin{array}{l}\text { 12. La Higiene en el servicio de urgencias es } \\
\text { agradable }\end{array}$ & $4 \%$ & $10 \%$ & $18 \%$ & $50 \%$ & $18 \%$ \\
\hline $\begin{array}{l}\text { 13. El servicio de urgencias cuenta con } \\
\text { personal de seguridad eficaz }\end{array}$ & $3 \%$ & $10 \%$ & $18 \%$ & $47 \%$ & $23 \%$ \\
\hline $\begin{array}{l}\text { 14. El personal de recepción atiende } \\
\text { oportunamente y con educación a los } \\
\text { usuarios }\end{array}$ & $4 \%$ & $9 \%$ & $26 \%$ & $46 \%$ & $15 \%$ \\
\hline
\end{tabular}




\begin{tabular}{|l|l|l|l|l|l|}
\hline \multicolumn{1}{|c|}{ Aspectos } & $\begin{array}{c}\text { Muy en } \\
\text { desacuerdo }\end{array}$ & $\begin{array}{c}\text { En } \\
\text { desacuerdo }\end{array}$ & \multicolumn{1}{|c|}{ Indeciso } & De acuerdo & \multicolumn{1}{c|}{$\begin{array}{c}\text { Muy de } \\
\text { acuerdo }\end{array}$} \\
\hline $\begin{array}{l}\text { 15. Las enfermeras auxiliares atienden } \\
\text { apropiadamente a los enfermos }\end{array}$ & $5 \%$ & $11 \%$ & $20 \%$ & $49 \%$ & $14 \%$ \\
\hline $\begin{array}{l}\text { 16. Las enfermeras Jefes coordinan } \\
\text { adecuadamente la atención de los usuarios }\end{array}$ & $5 \%$ & $11 \%$ & $31 \%$ & $37 \%$ & $16 \%$ \\
\hline $\begin{array}{l}\text { 17. Los médicos están capacitados para } \\
\text { atender enfermedades urgentes }\end{array}$ & $6 \%$ & $8 \%$ & $26 \%$ & $39 \%$ & $20 \%$ \\
\hline $\begin{array}{l}\text { 18. El médico lo escucha con atención antes } \\
\text { de hacer el diagnostico }\end{array}$ & $4 \%$ & $9 \%$ & $18 \%$ & $45 \%$ & $25 \%$ \\
\hline $\begin{array}{l}\text { 19. El médico lo examina detalladamente } \\
\text { antes de hacer el diagnostico }\end{array}$ & $5 \%$ & $13 \%$ & $16 \%$ & $39 \%$ & $27 \%$ \\
\hline $\begin{array}{l}\text { 20. El médico le solicita exámenes médicos } \\
\text { para un buen diagnóstico en caso de ser } \\
\text { necesario }\end{array}$ & $4 \%$ & $10 \%$ & $15 \%$ & $40 \%$ & $32 \%$ \\
\hline
\end{tabular}

Fuente. Elaboración propia.

A continuación se muestran las figuras más relevantes de acuerdo a las respuestas dadas por los usuarios encuestados.

Figura 3. El servicio de urgencias tiene capacidad física suficiente para la atención de la población de Yopal.

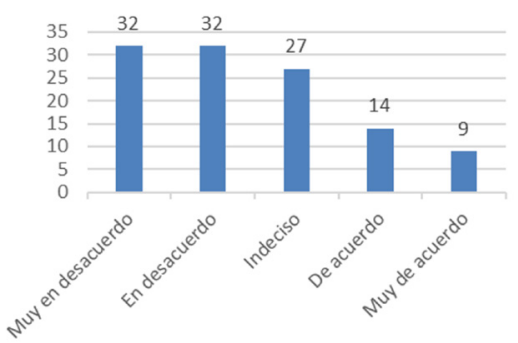

Fuente. Elaboración propia.

A la pregunta: ¿El servicio de urgencias tiene capacidad física suficiente para la atención de la población de Yopal?, el $28 \%$ respondió estar muy en desacuerdo y otro $28 \%$ respondió estar en desacuerdo.

Figura 4. La cantidad de personal en atención de urgencias es el apropiado.

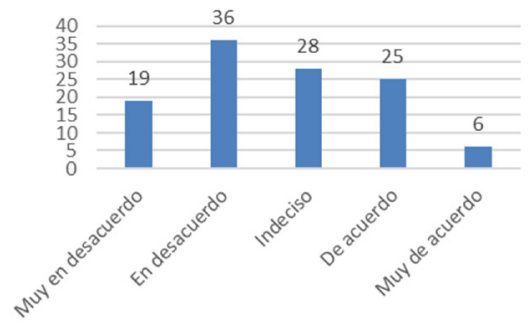

Fuente. Elaboración propia.
A la pregunta: ¿La cantidad de personal en atención de urgencias es el apropiado?, el $32 \%$ respondió estar en desacuerdo y el $25 \%$ respondió estar indeciso.

Figura 5. El personal de atención tiene preferencias sobre algunos pacientes.

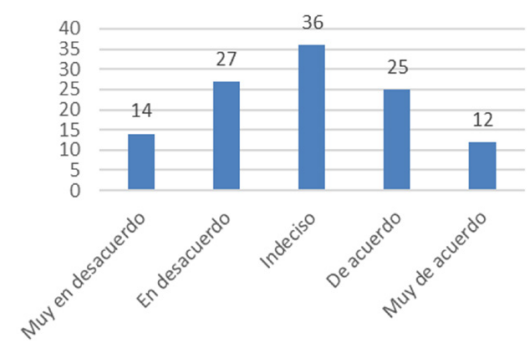

Fuente. Elaboración propia.

A la pregunta: ¿El personal de atención tiene preferencias sobre algunos pacientes?, el $32 \%$ respondió estar indeciso y el $24 \%$ respondió estar en desacuerdo.

Figura 6. La atención en la recepción de urgencias es excelente.

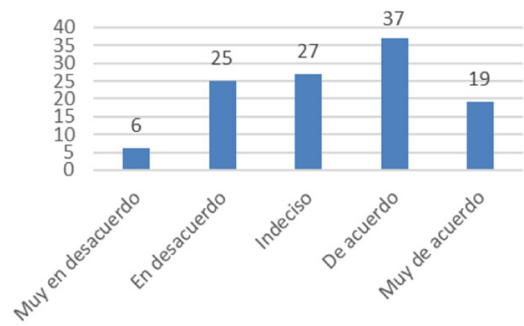

Fuente. Elaboración propia. 
A la pregunta: ¿La atención en la recepción de urgencias es excelente?, el $32 \%$ respondió estar de acuerdo y el $24 \%$ respondió estar indeciso.

Figura 7. El tiempo de atención en urgencias es suficiente y apropiado.

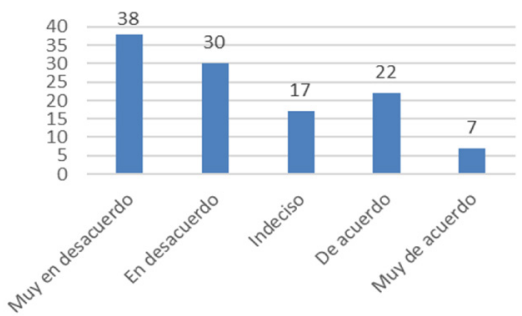

Fuente. Elaboración propia.

A la pregunta: ¿El tiempo de atención en urgencias es suficiente y apropiado?, el $33 \%$ respondió estar muy en desacuerdo y el $26 \%$ respondió estar en desacuerdo.

Figura 8. La sala de espera del servicio de urgencias es cómoda.

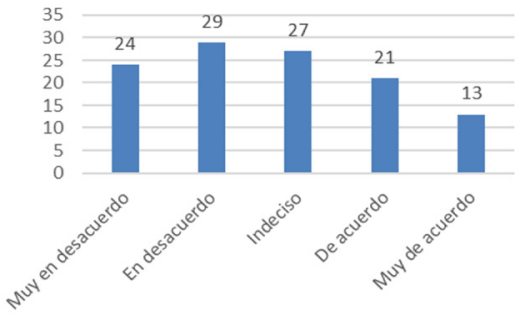

Fuente. Elaboración propia.

A la pregunta: ¿La sala de espera del servicio de urgencias es cómoda?, el 25 \% respondió estar en desacuerdo y el $24 \%$ respondió estar indeciso.

El análisis de las encuestas realizadas arroja unos porcentajes de desacuerdo o insatisfacción por parte de los usuarios, que vendrían a ser los puntos a destacar o atacar.

\section{Resultados de inconformidad}

Pregunta 3. ¿El servicio de urgencias tiene capacidad física suficiente para la atención de la población de Yopal?

Resultado: El 28 \% está en total desacuerdo y otros $28 \%$ en desacuerdo, que sumados arrojan un $56 \%$ de inconformidad.
Análisis: El servicio de urgencias del Hospital público de Yopal no tiene capacidad para atender la población del municipio, adicional a ello los usuarios de municipios aledaños o vecinos acuden a la capital del departamento (El Yopal) buscando una mejor atención, ya que solo tienen centros de atención y no cuentan con tecnología y personal especializado.

Pregunta 4. ¿La cantidad de personal en atención de urgencias es el apropiado?

Resultado: El $32 \%$ está en desacuerdo y el $17 \%$ en total desacuerdo, que sumados arrojan un $49 \%$ de inconformidad.

Análisis: Es preocupante la falta de personal profesional para la atención en el servicio de urgencias de Yopal, las largas jornadas de espera de los usuarios enfermos por falta de atención generan un ambiente desagradable y en algunos casos se torna violento.

Pregunta 5. El personal de atención tiene preferencias sobre algunos pacientes.

Resultado: El $32 \%$ está de acuerdo y el $11 \%$ muy de acuerdo, que sumados arrojan un $43 \%$ de inconformidad por la imparcialidad o igualdad que debería existir por ética.

Análisis: Algunos usuarios se quejan de existir preferencias de atención en algunos casos, sin que sean estos críticos o muy urgentes. Un servicio de calidad debe ser imparcial con los usuarios.

Pregunta 8. ¿El tiempo de atención en urgencias es suficiente y apropiado?

Resultado: El $33 \%$ está muy en desacuerdo y los 26 $\%$ en desacuerdo, que sumados arrojan un $59 \%$ de desconcierto o insatisfacción.

Análisis: Los usuarios se sienten inconformes con el tiempo que le dedican los profesionales en el momento de la atención. Ellos consideran que es muy corto el tiempo para realizar el diagnóstico y formular los medicamentos o tratamientos a seguir. Algunos pacientes se quejan de no recibir atención con calidad ya que por la premura no los escuchan con atención, no los examinan detalladamente ni les ordenan los exámenes médicos necesarios para un buen diagnóstico. 
Pregunta 9. La sala de espera del servicio de urgencias es cómoda

Resultado: El 25 \% está en desacuerdo y el $21 \%$ en total desacuerdo que sumados arrojan un $46 \%$ de inconformidad.

Análisis: Los usuarios se sienten incomodos en el área o espacio de espera en el servicio de urgencias, la alta demanda de enfermos en tan corto espacio genera una alta densidad de personal con sus consecuencias de acaloramiento y mal ambiente. Yopal necesita un hospital público con un espacio más grande para poder ofrecer una sala de espera más cómoda y con mejores condiciones.

\section{Discusión}

En las respuestas dadas por los usuarios encuestados se destacan las preguntas 3, 4, 5, 8 y 9 por tener un alto grado de inconformidad o insatisfacción. Los usuarios resaltan la inconformidad en los siguientes aspectos:

1. El personal profesional para la atención en el servicio de urgencias de Yopal no es suficiente para la alta demanda de enfermos.

2. La planta física del servicio de urgencias no tiene la capacidad suficiente para la alta demanda de los usuarios de Yopal y los municipios vecinos.

3. El tiempo de atención para realizar el diagnóstico y formular los medicamentos o tratamientos a seguir es muy corto.

4. La sala de espera es muy pequeña para la gran cantidad de usuarios que llegan a diario.

5. El personal de atención tiene preferencias en la atención sobre algunos pacientes.

Se radicó un documento en la oficina de calidad del hospital de Yopal solicitando información de algunos documentos para verificar o contrarrestar la respuesta de los encuestados pero la respuesta fue negativa, argumentando que por razones internas no podían brindar tal información. Los documentos solicitados fueron los siguientes:
1. Información general del servicio de urgencias, capacidad instalada.

2. Protocolos de atención.

3. Protocolos de calidad e indicadores.

4. Rutas de atención del servicio.

Se logró obtener información del hospital de Yopal a través del documento "Plan de gestión gerencial hospital de Yopal E.S.E. (2012)”. Recuperado en fuentes de Internet.

El sector salud en Colombia actualmente está en crisis, sin embargo, en Yopal se presenta una situación grave que hace más compleja y crítica la problemática. Desde el año 2011, Yopal no tiene agua potable es sus viviendas, la empresa de acueducto sufrió un derrumbe por falla geológica y a la fecha presente no existe una solución. Es muy alta la demanda en servicios de salud de urgencias para población pediátrica por enfermedades infecciosas.

La Secretaría de Salud de Yopal continúa realizando vigilancia de la calidad del agua para consumo humano. "La Administración Municipal de Yopal a través de la Secretaría de Salud como autoridad sanitaria responsable de vigilar la calidad del Agua para Consumo Humano, adelanta la recolección de toma de muestras de agua en los diferentes sistemas de abastecimiento, tanto urbana como rural, del municipio de Yopal” (26).

Existe dificultad en la accesibilidad a los servicios de tercer nivel de complejidad como necesidad de procedimientos de referencia y contra referencia, lo cual ocasiona estancias prolongadas en los servicios de urgencias.

\section{Conclusiones}

El hospital de Yopal no tiene capacidad suficiente en el área de urgencias para atender la alta demanda proveniente de los habitantes de Yopal y los municipios aledaños. Existe una constante queja de los usuarios que dicen ser mal atendidos, y el supuesto 
incumplimiento en su labor hacia los enfermos y familiares en la mayoría de las áreas del hospital agrava la situación.

Falta de personal profesional para la atención en el servicio de urgencias de Yopal, las largas jornadas de espera de los usuarios enfermos por falta de atención generan un ambiente desagradable y en algunos casos se torna violento

Los usuarios se sienten inconformes con el tiempo que le dedican los profesionales en el momento de la atención. Ellos consideran que es muy corto el tiempo para realizar el diagnóstico y formular los medicamentos o tratamientos a seguir. Algunos pacientes se quejan de no recibir atención con calidad ya que por la premura no los escuchan con atención, no los examinan detalladamente ni les ordenan los exámenes médicos necesarios para un buen diagnóstico.

Los usuarios se sienten incomodos en el área o espacio de espera en el servicio de urgencias, la alta demanda de enfermos en tan corto espacio genera una alta densidad de personal con sus consecuencias de acaloramiento y mal ambiente. Yopal necesita un hospital público con un espacio más grande para poder ofrecer una sala de espera más cómoda y con mejores condiciones.

\section{Recomendaciones}

De acuerdo al análisis cuantitativo y cualitativo realizado a las encuestas que se aplicaron a los usuarios del servicio de urgencias del hospital público de Yopal y la aplicación de la técnica de observación directa, análisis e interpretación de fenómenos, acontecimientos y hechos que prevalecen en el área se dan las siguientes recomendaciones:

1) La administración del municipio de Yopal debe promover y gestionar la ampliación del hospital público de Yopal o la construcción y puesta en marcha de clínicas privadas o entidades de salud.
2) La gobernación del Casanare debe gestionar la optimización de los centros de salud con tecnología, personal profesional calificado y buena atención en las veredas y municipios del Casanare.

3) La secretaría de salud del municipio de Yopal debe seguir vigilando la calidad del agua para consumo humano y seguir haciendo campańas de salud para evitar el consumo de agua no potable.

4) La administración del hospital de Yopal E.S.E debe orientar su gestión en pro de fortalecer el sistema de gestión de calidad con el fin de prestar servicios de salud óptimos enfocados en el marco de la seguridad del paciente, dando cumplimiento a las normas que respaldan a la calidad total.

5) La oficina de calidad del hospital público de Yopal debe estar vigilante en la calidad de la atención, cumplir con la normatividad según el marco del Sistema General de Seguridad Social en Salud (SGSSS) y la normatividad vigente, así como la provisión de servicios de salud a los usuarios de forma accesible, equitativa y con un nivel profesional óptimo.

6) Los profesionales del servicio de urgencias deben cumplir con los protocolos de calidad y aplicar el buen uso de "Las Guías para Manejo de Urgencias". Es de vital importancia que los médicos, enfermeras, y demás profesionales de la salud, tengan los conocimientos, habilidades y destrezas necesarias para poder diagnosticar y manejar de la mejor manera, las urgencias médicas que se puedan presentar.

7) Proponer como estrategia gerencial a la administración del hospital de Yopal E.S.E, el diseño y la aplicación de mecanismos de control de calidad enfocados en la política de humanización de la atención, con la implementación de procesos de formación continua a los profesionales del servicio de urgencias con el fin de proporcionar un servicio solidario, tolerante y de respeto a los derechos de los pacientes, un servicio expresado primero en la calidad del profesional de salud como ser humano en su atención al paciente. 


\section{Referencias}

1. Creswell, J. W. Research Design: qualitative, quantitative, and mixed methods approaches, Londres: Sage; 2005.

2. Briceño C. Modelo para evaluar calidad del servicio en una IPS de alta complejidad, en su componente de "satisfacción del usuario”. UNAB. 2001; 2(3): 1-9. Recuperado de: http:// revistas.unab.edu.co/index.php?journal $=$ marketing\&page $=$ article\&op=viewArticle\&path $\% 5 B \% 5 \mathrm{D}=758$

3. Garcia CM. El Hospital como empresa: nuevas prácticas, nuevos trabajadores. Universidad Piloto de Colombia. 2007; 6 (1): 143-154.

4. Ministerio de Salud. Guías para manejo de urgencias. Tomo I. Recuperado de: https://www.minsalud.gov.co/Documentos\%20y\%20Publicaciones/Gu\%C3\%ADa\%20para\%20manejo $\% 20$ de $\% 20$ urgencias $\% 20$-Tomo\%20I.pdf

5. Gustavo A, Cabrera-Arana LD, Bello-Parías, Londoño-Pimienta JL. Calidad Percibida por Usuarios de Hospitales del Programa de Reestructuración de Redes de Servicios de Salud de Colombia. Rev. Salud pública. 2008; 10 (4): 593-604.

6. Hernández Sampieri FC, Baptista L. Metodología de la investigación. México: McGraw-Hill; 2010.

7. Hospital de Yopal. [citado 5 de julio]. Recuperado de: http:// hospitaldeyopal.gov.co/es/inicio

8. Kerguelén Botero CA. Calidad en salud en Colombia, Los principios. Ministerio de la Protección Social Programa de Apoyo a la Reforma de Salud, PARS. Colombia: Editorial Scripto Ltda; 2008.

9. Hospital de Yopal. Manual de inducción y reinducción, hospital de Yopal ESE. Recuperado de: http://hospitaldeyopal. gov.co/apc-aa-files/37326365336631663061623632646336/ manual-de-reindiccion-final-nov-2014.pdf

10. Manual de información y atención al usuario SIAU. Recuperado de: http://www.redoriente.net/siscalidad/3.pdf

11. Massip C, Ortiz R, Llantá M, Peña M; Infante I. La evaluación de la satisfacción en salud: un reto a la calidad. Rev Cubana Salud Pública. 2008; 34 (4).

12. Ministerio de salud y protección social. Calidad de atención en salud. [citado 6 de julio del 2016]. Recuperado de: https://www.minsalud.gov.co/salud/CAS/Paginas/calidad-atencion-en-salud-home.aspx

13. Ministerio de Salud. Nuevos criterios para clasificación de triage en urgencias. [citado 6 de julio del 2016]. Recuperado de: https://www.minsalud.gov.co/Paginas/Nuevos-criterios-para-clasificaci\%C3\%B3n-de-triage-en-urgencias.aspx

14. Ministerio de Salud. Observatorio de calidad en la atención en salud. Recuperado de: http://calidadensalud.minsalud.gov.co/ Observatorio.aspx

15. Plan de gestión gerencial hospital de Yopal E.S.E. 2012. Recuperado de: http://www.hospitaldeyopal.gov.co/apc-aafiles/37326365336631663061623632646336/plan-de-gestion-2012.pdf
16. República de Colombia. Política Nacional de Prestación de Servicios de Salud. Bogotá: Ministerio de la Protección Social; 2005. Recuperado de: https://www.minsalud.gov.co/Ministerio/Documents/Politica\%20Nacional\%20de\%20Prestaci\%C3\%B3n\%20de\%20Servicios\%20de\%20Salud.pdf

17. Ross A, Zeballos J, Infante A. La calidad y la reforma del sector salud en América Latina y el Caribe. RevPanam Salud Publica. 2000; 8 (1,2): 93-98.

18. Riveros J, Berné C. Análisis de la opinión de usuarios sobre calidad percibida y satisfacción con hospitales públicos: Estudio de caso desde la perspectiva de la aplicación del marketing. Rev. Méd. Chile. 2007; 135: 862-870.

19. Bernal LM, López G. Diagnóstico prénatal: retrospectiva. Nova. 2014; 12( 21 ): 23-36.

20. Márquez MA, Gómez GM. Accidente ofídico en el departamento de Sucre, Colombia. Nova. 2015; 13( 24 ): 39-46.

21. González YL. Evaluación de la percepción del riesgo ocupacional en trabajadores de una empresa del sector de la construcción en Bogotá D.C. Nova. 2015; 13 ( 23 ): 93-107.

22. Carrero SH, Heredia Montoya DP, Bolaños YM, Medellín Martín Orlando Pulido. Seroprevalencia de infección por Leptospira y factores de riesgo en estudiantes de una universidad de Colombia. Nova. 2017; 15( 27 ): 131-138.

23. Naranjo RA. Avances y perspectivas en Síndrome de Asperger. Nova. 2014; 12( 21 ): 81-101.

24. Almonacid CC, Camarillo Romero MS, Gil Murcia Z, Medina Medina CY, Rebellón Marulanda JV, Mendieta Zerón H. Evaluación de factores de riesgo asociados a enfermedad cardiovascular en jóvenes universitarios de la Localidad Santafé en Bogotá, Colombia. Nova. 2016; 14(25): 9-17.

25. González JL., Monroy Romero PA., Almonacid Urrego CC. Homocisteína y otros factores de riesgo cardiovascular en niños de educación básica primaria del Colegio Distrital Manuel Elkin Patarroyo, Bogotá, D.C.Colombia. Estudio piloto. Nova. 2017; 15 ( 27 ): 103-117.

26. Llanera.com. Secretaria de salud de Yopal continúa monitoreando calidad del agua para consumo humano; 2015. Recuperado de: https://llanera.com/secretaria-de-salud-de-yopal-continua-monitoreando-calidad-del-agua-para-consumo-humano/21224 